\title{
Molecular Alignment of a Meta-Aramid on Carbon Nanotubes by In-Situ Interfacial Polymerization.
}

Cécile A. C. Chazot, * Behzad Damirchi, Adri C. T. van Duin, A. John Hart*

Cécile A. C. Chazot - Department of Materials Science and Engineering, Department of Mechanical Engineering, Massachusetts Institute of Technology, 77 Massachusetts Avenue, Cambridge, Massachusetts 02139, United States.

Behzad Damirchi - Department of Mechanical Engineering, Pennsylvania State University, 245 Research East, University Park, Pennsylvania, 16802, United States. †

Adri C. T. Van Duin - Department of Mechanical Engineering, Pennsylvania State University, 240 Research East, University Park, Pennsylvania, 16802, United States.

A. John Hart - Department of Mechanical Engineering, Massachusetts Institute of Technology, 77 Massachusetts Avenue, Cambridge, Massachusetts 02139, United States.

\section{KEYWORDS}

Molecular Dynamics, Carbon nanotubes, Polymers, Interfacial Polymerization, Nucleation
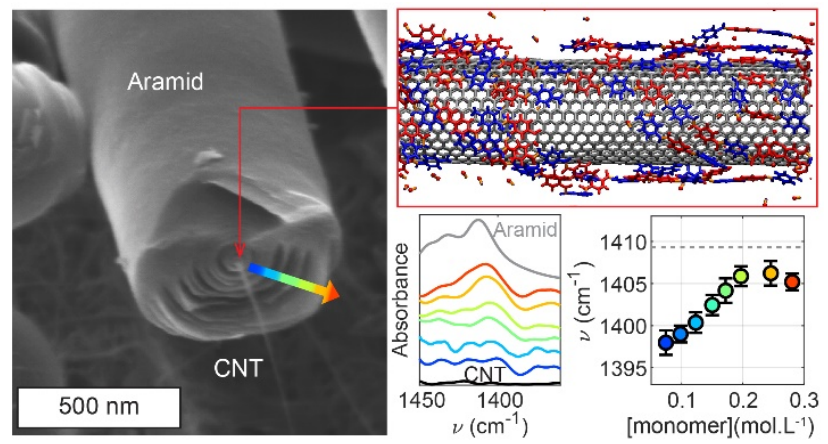


\begin{abstract}
Molecularly organized nanocomposites of polymers and carbon nanotubes (CNTs) have great promise as high-performance materials; in particular, conformal deposition of polymers can control interfacial properties for mechanical load transfer, electrical or thermal transport, or electro/chemical transduction. However, controllability of polymer-CNT interaction remains a challenge with common processing methods that combine CNTs and polymers in melt or in solution, often leading to non-uniform polymer distribution and/or aggregation of CNTs. Here, we demonstrate CNTs within net-shape sheets can be controllably coated with a thin, conformal coating of meta-aramid by simultaneous capillary infiltration and interfacial polymerization. We determine that $\pi$ interaction between the polymer and CNTs results in chain alignment parallel to the CNT outer wall. Subsequent nucleation and growth of the precipitated aramid forms a smooth continuous layered sheath around the CNTs. These findings motivate future investigation of mechanical and interfacial properties of the resulting CNT composites, and adaptation of the insitu polymerization method to other substrates.
\end{abstract}

\title{
INTRODUCTION
}

Ordered polymer nanocomposites provide a route to high-performance lightweight structural materials with applications including aircraft and spacecraft structures, sporting equipment, and filtration membranes. ${ }^{1}$ Research and development on nanocomposite processing has been devoted to the combination of fillers such as high-crystallinity natural ${ }^{2,3}$ or synthetic $\mathrm{c}^{4,5}$ polymer fibers or carbon nanotubes $(\mathrm{CNTs})^{6,7}$ with polymers such as poly(vinyl alcohol $)^{3,8,9}$ or commercial resin systems. ${ }^{10}$ The molecular organization and potential ordering of the polymer at the surface of the nanofillers has also been a particular center of attention as a mean to affect overall composite performance. ${ }^{6}$

Due to their outstanding mechanical and electrical properties and low mass density, the composites of CNTs with engineered polymers offer great potential as next-generation lightweight structural materials. ${ }^{11}$ CNT-polymer composites have most commonly been made by resin infiltration of CNT mats or fibers, thereby translating manufacturing techniques widely used for carbon-fiber composites. ${ }^{6}$ Nevertheless, as the size of the reinforcement fibers reduces to the nanoscale, the complex interplay among transport-limited polymer infiltration, curing kinetics, and CNT-polymer molecular interaction greatly impacts the local polymer content and properties, and 
therefore mechanical properties of the final composites. For example, the polymer chemistry and CNT surface chemistry can greatly influence polymer chain packing and organization, through localized chain alignment, ${ }^{12}$ wrapping around the $\mathrm{CNTs}^{13}$ or even long-range crystallization. ${ }^{7,9}$ The chemical interaction between the polymer and CNTs also affects wetting of the resin precursor on the CNTs, the resin infiltration speed, ${ }^{10,14}$ polymerization or curing reaction kinetics, ${ }^{15}$ and final composite mechanical properties through load transfer. ${ }^{16}$ However, the multi-component nature of the polymer precursors impedes the assessment of each compound's contribution to the resin-CNT interaction. This intricacy constitutes a major challenge to experimentally understand and accurately compute the influence of CNTs on polymer formation. The study of such complex systems also prevents the extraction of guiding principles towards the design of polymer systems specifically for high-performance CNT-based composites. Moreover, resin cure cycles typically involve high temperature treatment for prolonged periods of times, from hours to days, ${ }^{17,18}$ making systematic studies of the CNT-polymer interaction particularly time- and energy-consuming.

Here we show that conformal polymer coatings on CNTs can be obtained by in-situ interfacial polymerization (ISIP) of meta-aramids, ${ }^{19,20}$ through short-range molecular ordering of the polymer chains and long-range nucleation and growth of polymer particles. ISIP of metaaramid poly(m-phenylene isophthalamide) (PMPI) relies on the reaction of m-phenylene diamine (MPD) dissolved in water with isophthaloyl chloride (IPC) dissolved in an organic solvent (in this case, cyclohexanone) (Figure 1). A nanoporous aligned CNT sheet (Figure 1a), obtained commercially and produced by floating-catalyst chemical vapor deposition (CVD), is immersed in the aqueous and organic solutions subsequently (Figure 1b,c). Upon contact with the organic phase, the liquid wicks into the network by capillary action, displacing the aqueous phase (Figure 1c). MPD is transported across the moving liquid-liquid interface into the organic phase where it immediately reacts with IPC, producing PMPI chains and releasing hydrochloric acid as a byproduct. As the PMPI chains grow in length, they precipitate out of the organic phase to form solid polymer onto the CNTs. Therefore, ISIP relies on the simultaneous liquid-liquid interface motion and polymer precipitation inside the nanoporous network of CNTs. Last, rinsing and solvent evaporation result in a CNT-PMPI composite free of impurities and unreacted monomers (Figure 1d). By adjusting monomer concentration, the mass of polymer produced as well as the morphology of the polymer coating onto the CNTs can be adjusted. ${ }^{20}$ 
a

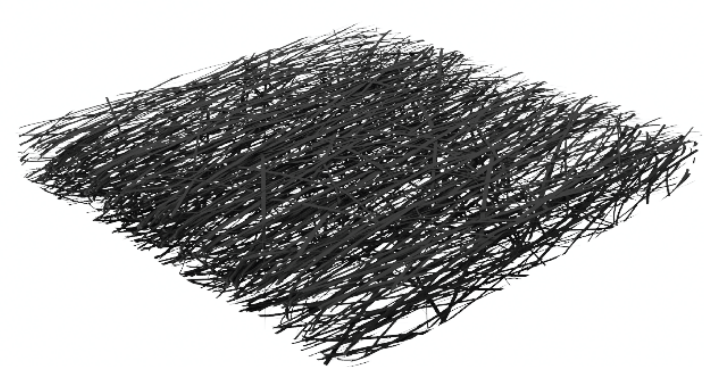

b

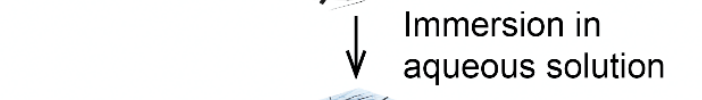

C
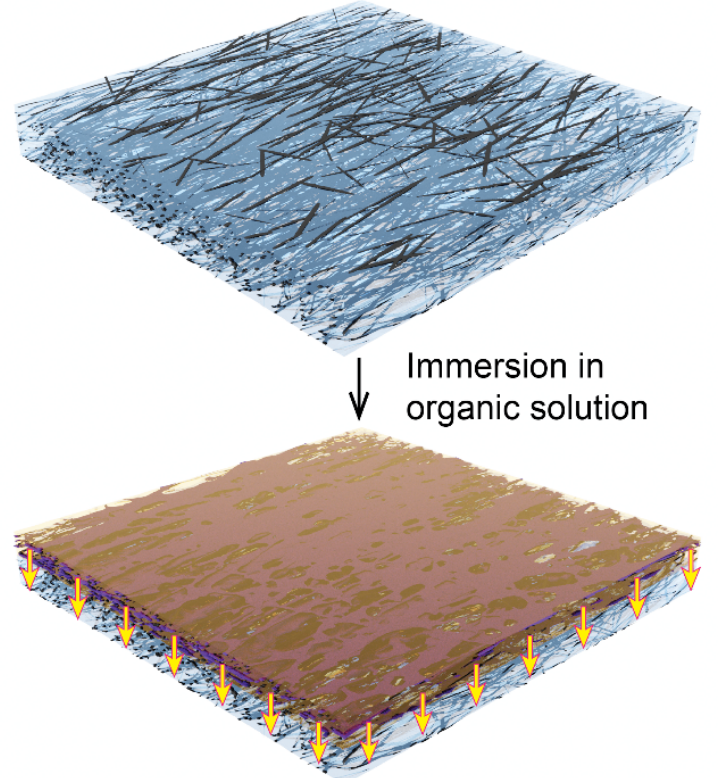

d

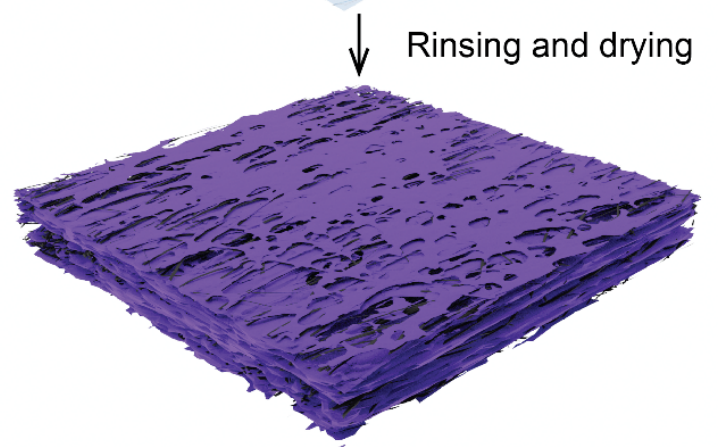

CNT

Diacyl choride solution

Diamine solution

Solid aramid

Figure 1. Schematic rendering of the ISIP process, forming a polymer-CNT composite sheet. (a) Neat aligned CNT mat. (b) CNT nanoporous network imbibed with water solution. (c) Immersion in the organic solution results in simultaneous liquid displacement and polymer formation on the organic side of the interface. (d) After rinsing and drying a CNT-aramid composite mat is obtained. 
In recent work, we showed that PMPI forms in droplets or conformal sheaths around the CNTs, depending on the relative rates of transport and reaction kinetics. ${ }^{20}$ The polymer formed is amorphous in nature, demonstrating that commonly observed crystallization of meta-aramids through interchain hydrogen interaction ${ }^{21}$ does not contribute to ordering of PMPI in the vicinity of CNTs. Instead, we expect from literature that the prominent $\pi$-interaction ${ }^{22}$ involving PMPI meta-phenyl rings and the outer wall of CNTs induces short-range molecular ordering of polymer chains onto the CNT. This sub-nanometer ordered polymer layer is hypothesized to serve as a template for further precipitation by heterogeneous nucleation of amorphous PMPI particles onto the CNTs, governing long-range polymer sheath formation.

\section{RESULTS AND DISCUSSION}

First, we aim to experimentally identify the governing phenomenon for the formation of conformal polymer sheaths. Precipitation kinetics and its dependence on monomer concentration in the ISIP process is assessed by scanning electron microscopy (SEM) observation of the polymer morphology in presence of CNTs (Figure 2). At low monomer concentrations $\left(0.098\right.$ mol. $\left.\mathrm{L}^{-1}\right)$, PMPI precipitates onto the CNT surface as nanometer-scale droplets and thin polymer sheaths (Figure 2a,b). For experiments performed at higher concentrations, from 0.098 mol.L $\mathrm{L}^{-1}$ to 0.248 mol. $\mathrm{L}^{-1}$, the polymer sheath diameter increases from $\sim 20 \mathrm{~nm}$ to $\sim 40 \mathrm{~nm}$ (Figure $2 \mathrm{~b}, \mathrm{c}$ ). Additionally, while some CNTs are observed to have smooth conformal sheaths, others feature bead-like deposits adhered to an underlying smooth layer (Figure a-c). Last, at high monomer concentration (greater than $0.2 \mathrm{~mol} \cdot \mathrm{L}^{-1}$ ), and in regions where the CNT density is low in the network, smooth conformal polymer coatings with thicknesses in the order of $100 \mathrm{~nm}$ with a layered structure can be observed around individual CNTs (Figure 2d). These observations are strong evidence that PMPI forms by heterogeneous nucleation and growth of polymer particles at the surface of the CNTs or on the already-formed polymer sheaths (Figure 2e). Initial polymer nuclei form around individual CNTs and, as more polymer is formed, these coalesce as a continuous sheath which serves as the surface for subsequent precipitation. The layered structure of the larger sheaths observed at high concentration is consistent with this interpretation. 

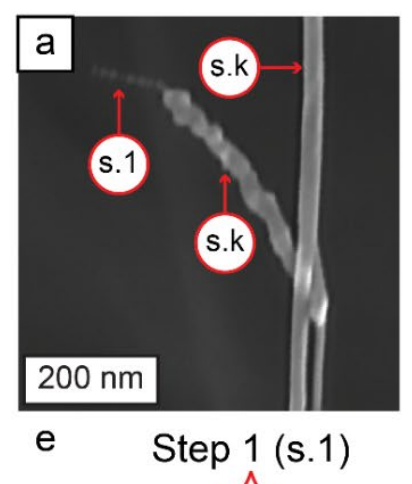
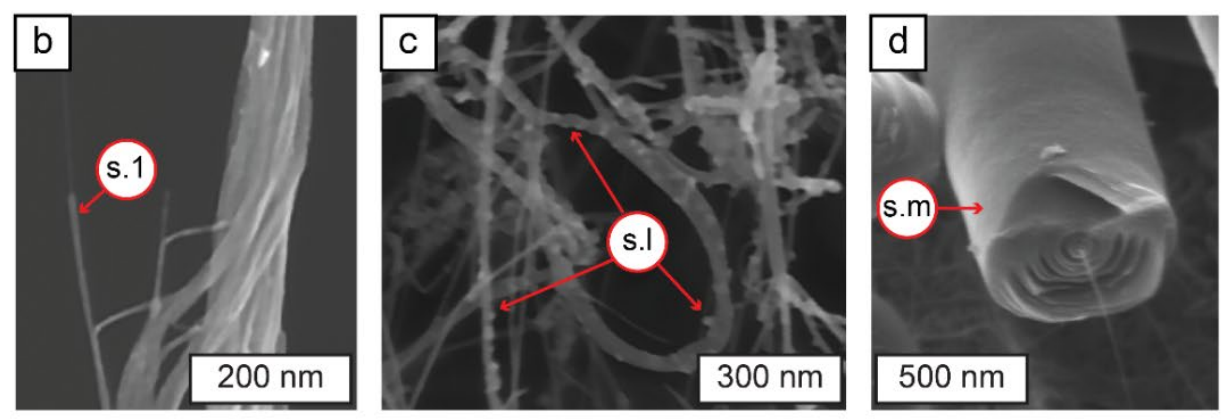

Step 2 (s.2)

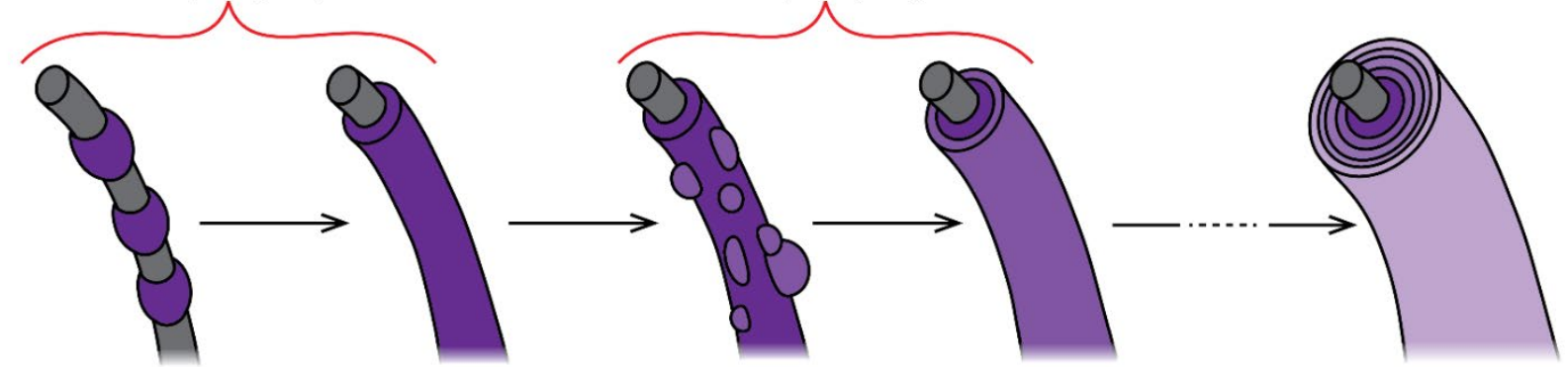

Figure 2. PMPI precipitation mechanism onto CNTs in ISIP. (a-d) SEM images of CNT-PMPI composites obtained for monomer concentrations of (a-b) 0.098 mol. $\mathrm{L}^{-1}$, (c) $0.248 \mathrm{mol.L^{-1 }}$ and (d) 0.281 mol.L . $^{-1}$ (e) Schematic describing the steps of nucleation and growth of PMPI onto CNTs and forming polymer sheaths. Nuclei form on a CNT before coalescing to form a smooth polymer sheath which subsequently supports polymer nuclei formation. Step 2 is repeated (s.x where $x \geq 2$ ) to form multiple polymer layers around the CNT. Specific steps of the polymer nucleation and growth process are also indicated on (a-d) where $k, l$ and $m$ are integers and $k<l<<m$.

The CNT-polymer chemical interaction and its influence on polymer ordering within the conformal sheaths is assessed by molecular dynamics (MD) simulations, as described in Supplementary Information section S1 (Figure 3). The reaction between MPD and IPC is assumed to occur in a thin reaction zone on the organic side of the cyclohexanone-water interface (Figure 3a). ${ }^{19}$ To assess $\pi-\pi$ stacking 22 between the surface of the CNTs and the aromatic rings of the PMPI backbone, we define the normal vector to each meta phenyl group of the polymer as the cross product of the two vectors generated using three nonadjacent aromatic carbon atoms (Figure $3 b$ ). The angle $\varphi$ between this normal vector and the normal to the CNT surface is used to calculate the Herman's orientation function $f^{23}$ which quantifies the alignment of the polymer aromatic rings in relation to the CNT surface: 


$$
f=\frac{3\left(\left\langle\cos ^{2} \varphi\right\rangle-1\right)}{2}
$$

Here the angle brackets stand for the average value over all angles, and

$$
\left\langle\cos ^{2} \varphi\right\rangle=\frac{\int_{0}^{\pi} \cos ^{2} \varphi \sin \varphi d \varphi}{\int_{0}^{\pi} \sin \varphi d \varphi}
$$

A value of $f=1$ describes parallel $\pi$ - $\pi$ stacking of all the aromatic rings with the CNT surface in a face-to-face or parallel staggered configuration, ${ }^{22}$ while a value of $f=0$ corresponds to random alignment of the polymer chains along the CNT surface. If $f=-0.5$, the vectors are perpendicular and the interaction of polymer chains with the CNTs can be described as $\pi-\pi \mathrm{T}$ shaped or $\pi-\pi$ Y-shaped stacking (also called edge-to-face orientation). ${ }^{22}$
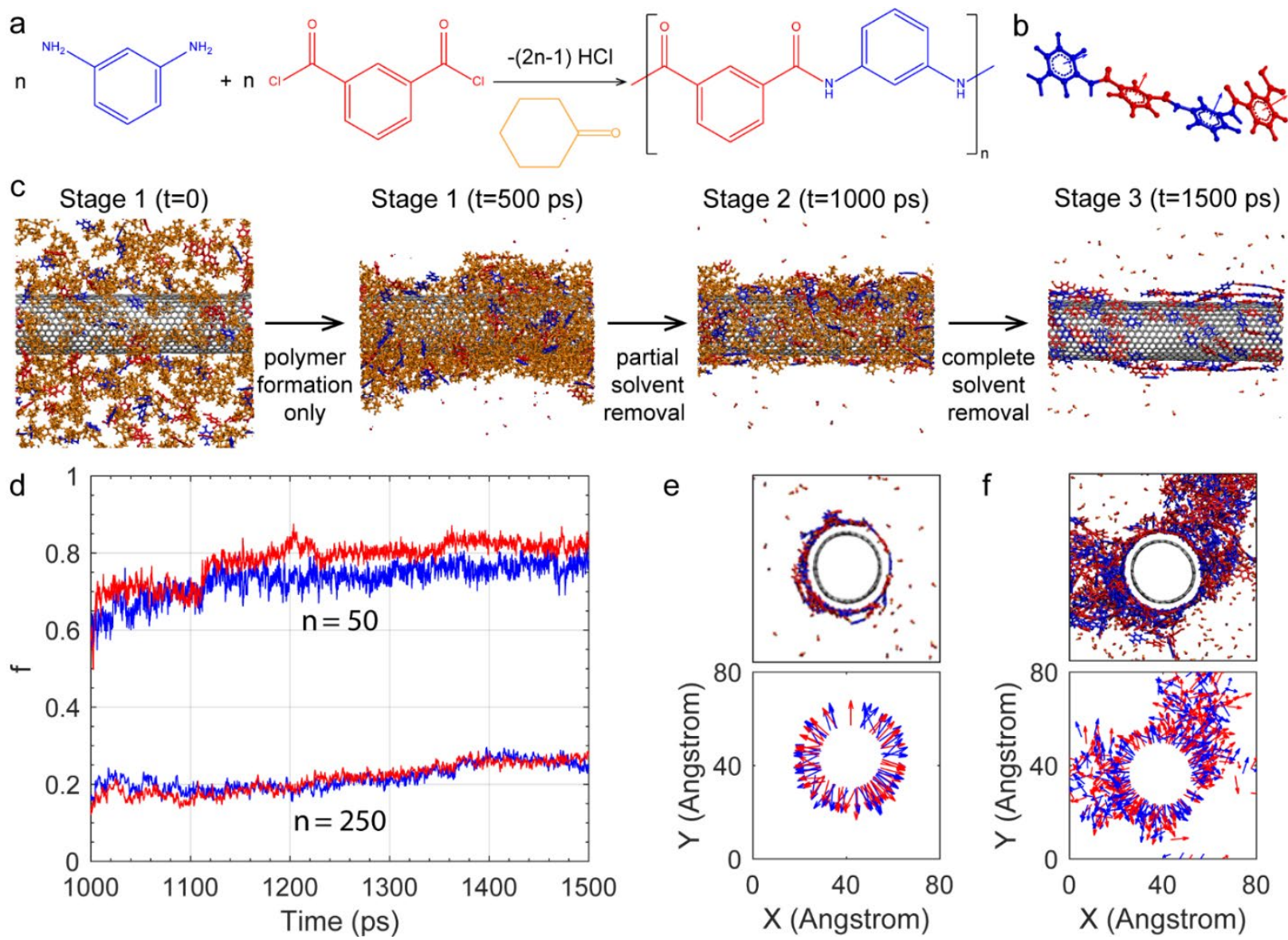

Figure 3. MD simulation of ISIP. (a) Polycondensation reaction for the formation of PMPI by interfacial polymerization. (b) Definition of the normal vector to the aromatic rings of the polymer backbone, here represented on a dimer $(n=2)$. (c) Snapshot at subsequent stages of the simulation 
showing monomer molecules attaching to each other and building oligomers, as well as half the solvent molecules begin removed (Stage 2), and then all the solvent molecules being removed (Stage 3). The color scheme is that of (a) and (b) and orange color represent cyclohexanone. (d) Herman's orientation function during stage 3 for a simulation featuring 50 and 250 of each monomer. Both simulations feature 500 cyclohexanone molecules. (e-f) Polymer morphology and spatial distribution of the normal vector to the PMPI aromatic rings at $t=1500 p$ s for an initial number of each monomer molecules of (e) 50 and (f) 250.

We use a ReaxFF reactive force field framework ${ }^{24}$ with chemical acceleration tools to enable reaction barrier sampling at time-scales accessible to ReaxFF-based molecular dynamics ${ }^{25-29}$. With this, we capture the polymerization reaction between MPD and IPC in the organic phase as well as the CNT-PMPI interaction. The simulation features three successive stages, shown in Figure 3c. The influence of monomer concentration on polymer-CNT interaction is studied by varying the number of monomer molecules (50 or 250 of each monomer) present in the system at $\mathrm{t}=0$ while keeping the initial number of cyclohexanone molecules constant and equal to 500 (Table $\mathrm{S} 1)$. During stage $1(0-500 \mathrm{ps})$, monomers are allowed to react with one another in presence of cyclohexanone molecules. Stage 2 and stage 3 (both 500ps in duration) correspond to removal of the organic solvent; at the end of stage 1, 250 cyclohexanone molecules are removed, and 250 more are removed between stage 2 and stage 3 (Table S1).

We monitor the value of the Herman's orientation function $f$ and the normal vector to the polymer chain aromatic groups in stage 3 (Figure $3 \mathrm{~d}$ ). At low concentration (50 of each monomer at $\mathrm{t}=0$ in the MD simulation), polymer chains form an ordered layer along the CNT surface (Figure $3 \mathrm{~d}$ ). With a $f$ value between 0.7 and 0.8 , the polymer chains' aromatic groups interact with the CNT outer wall via parallel $\pi-\pi$ stacking, resulting in parallel alignment of the polymer chains with the CNT surface (Figure 3e). With increasing monomer concentration (250 of each monomer at $\mathrm{t}=0), f$ is only $0.2-0.3$, and the PMPI chains are randomly aligned in the absence of $\pi-\pi$ stacking away from the CNTs. This low $f$ value is consistent with the experimental observation that PMPI synthesized by ISIP is amorphous and show no visible long-range order. Nevertheless, the spatial distribution of the normal vector to the aromatic rings of the polymer backbone (Figure 3f) indicates that chains located in the vicinity of the CNT still align along the surface of the nanotube via parallel $\pi-\pi$ stacking, while chains closer to the edge of the box form randomly aligned bridge 
between CNTs, as captured by periodic boundary conditions in the simulation. It is worth noting that experimental observation of sheath formation was performed on a mixture of cylindrical and self-collapsed (i.e., flattened) CNTs. Indeed, large-diameter single- and douhle-walled CNTs produced by CVD show collapsed geometry due their radial flexibility ${ }^{30-32}$ or defect density. ${ }^{33}$ Importantly, the MD simulation predicts the same alignment trend for collapsed CNTs (Figure S1).

Now, we experimentally evaluate the nature of the CNT-polymer molecular interaction and its evolution as PMPI forms increasing number of sheath layers around the CNTs. We combine Fourier transform infrared (FT-IR) and Raman spectroscopy, which together let us identify the vibrational modes affected by the CNT-polymer interaction. ${ }^{34,35}$

FT-IR spectroscopy is used first as PMPI exhibit a strong FT-IR signal while CNTs feature vibrational modes which are only Raman-active for the most part (Figure 4). ${ }^{35}$ This results in the straightforward isolation of the contribution from PMPI to the FT-IR spectrum, and its comparison against the neat polymer and neat CNT signals (Figure 4a). The detailed peak assignment for the FT-IR signature of PMPI can be found in Table S2. ${ }^{34,36}$ To analyze the evolution of PMPI-CNT $\pi$ interaction with increasing polymer content, we observe the location of selected peaks characteristic of in-plane (Figure 4b,c) and out-of-plane (Figure 4d) vibrational modes relevant to the meta-aromatic groups and the secondary amines of PMPI. For CNT-PMPI composites, the location of peaks representing PMPI meta-phenyl ring semicircle stretch mode (Figure 4b) and inplane C-H bend-stretching mode (Figure 4c) noticeably shift towards higher wavenumber with increasing monomer concentrations. For composites obtained with monomer concentrations greater $0.2 \mathrm{~mol} . \mathrm{L}^{-1}$, the peak location stabilizes at a value slightly smaller than that found on the neat PMPI FT-IR spectrum. The blue shift and asymptotic behavior of the in-plane aromatic vibrational peaks suggest that in-plane motion of the PMPI meta-phenyl ring is hindered when the polymer forms in the vicinity of the CNT outer wall, and that this hindrance decays as more polymer is formed, further from the CNT surface. Additionally, splitting of the peaks relevant to the two vibrations involved in semicircle stretch is observed for PMPI formed in presence of CNTs. The observed peak shift and splitting are consistent with a strong $\pi-\pi$ interaction ${ }^{35,37,38}$ that weakens with increasing polymer content as predicted by the MD simulation. Indeed, the strong $\pi$ interaction between the CNTs and polymer repeat units results in an attractive electrostatic force that disrupts the degrees of freedom involved in PMPI's characteristic vibrational modes. In the 
case of parallel $\pi-\pi$ stacking for instance, the electrostatic quadrupole interactions between $\pi$ orbitals of the CNT outer wall and PMPI aromatic groups results is disruption of the motion of the atoms involved in the in-plane vibrational modes, therefore affecting the frequency and relative intensity of the characteristic peaks.

Additional evidence of $\pi$ interaction can be found in the FT-IR peaks relevant to out-of-plane vibrational modes (Figure 4d). First, red-shift of the Amide $\mathrm{V}$ band provides evidence of interaction between the CNTs and the electron-rich -NH- group. This interaction appears to decrease rapidly with increasing thickness of the polymer sheath, as the peak position reaches a steady value of $721 \mathrm{~cm}^{-1}$ for monomer concentrations greater than $0.1 \mathrm{~mol} . \mathrm{L}^{-1}$, a value $5 \mathrm{~cm}^{-1}$ greater than that of neat PMPI. While the Amide V band shows this red-shift, in-plane vibrational modes related to the polymer amide linkage, such as Amide I and II vibrations corresponding to stretching of $\mathrm{C}=\mathrm{O}$ and $\mathrm{C}-\mathrm{N}$ bonds respectively, show no significant frequency shift. This can be explained by an additional NH- $\pi$ interaction ${ }^{22,35,38}$ between PMPI and CNTs. The red-shift of the Amide $\mathrm{V}$ band occurs in a narrower range of concentrations compared to the aromatic parallel vibrational modes. This reflects the weaker interaction energy involved in NH- $\pi$ compared to $\pi-\pi$ stacking. ${ }^{38}$ Moreover, the FT-IR peak corresponding to the meta-phenyl group's sextant out-ofplane bending mode shows a concentration-independent blue shift of $10 \mathrm{~cm}^{-1}$ compared to neat PMPI. The combination of concentration-dependent peak location for in-plane aromatic vibrational modes with concentration-independent significant peak shift for out-of-plane aromatic vibrational modes hints at parallel $\pi-\pi$ stacking between CNTs and PMPI. In edge-to-face $\pi$ interaction, one positively charged structure of each PMPI ring is juxtaposed against the $\pi$ electron cloud of the CNT ring, ${ }^{6,22}$ leaving most of the other bonds free to vibrate, particularly through inplane motions. On the other hand, in parallel $\pi-\pi$ stacking, multiple aromatic sub-groups in each PMPI ring feature electrostatic interaction with the $\pi$ electron cloud of the CNT outer wall. Parallel $\pi$ - $\pi$ stacking results in greater restraint of both the in-plane and out-of-plane aromatic bonds' vibration, therefore altering the shape of the relevant FT-IR peaks. 

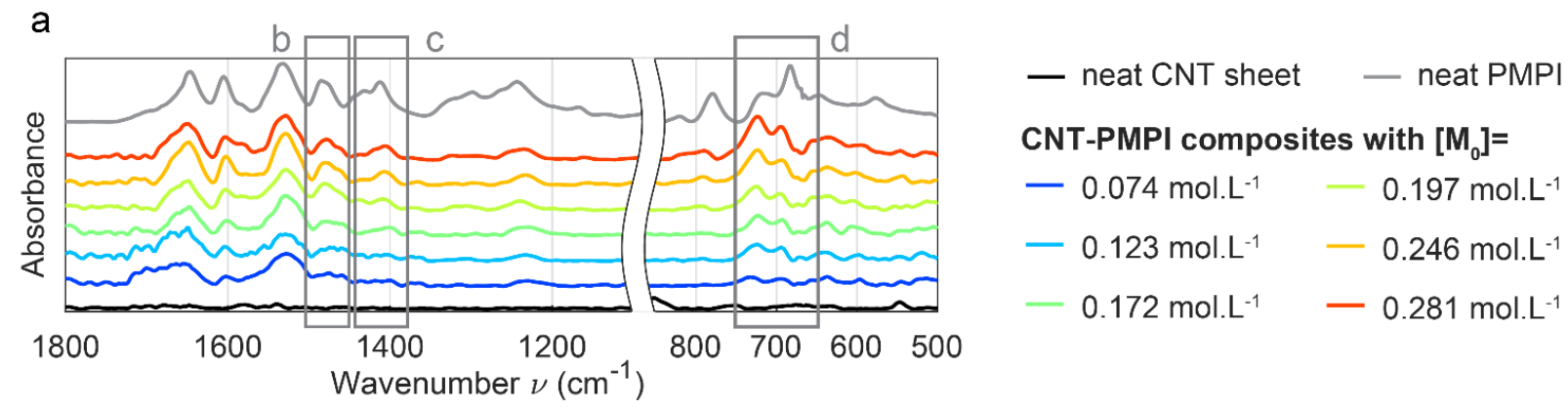

$-0.074 \mathrm{~mol}^{-\mathrm{L}^{-1}}-0.197 \mathrm{~mol}^{-\mathrm{L}^{-1}}$

$0.123 \mathrm{~mol} . \mathrm{L}^{-1} \quad-0.246 \mathrm{~mol}^{-1} \mathrm{~L}^{-1}$

$0.172 \mathrm{~mol} . \mathrm{L}^{-1} \quad-0.281 \mathrm{~mol} \cdot \mathrm{L}^{-1}$
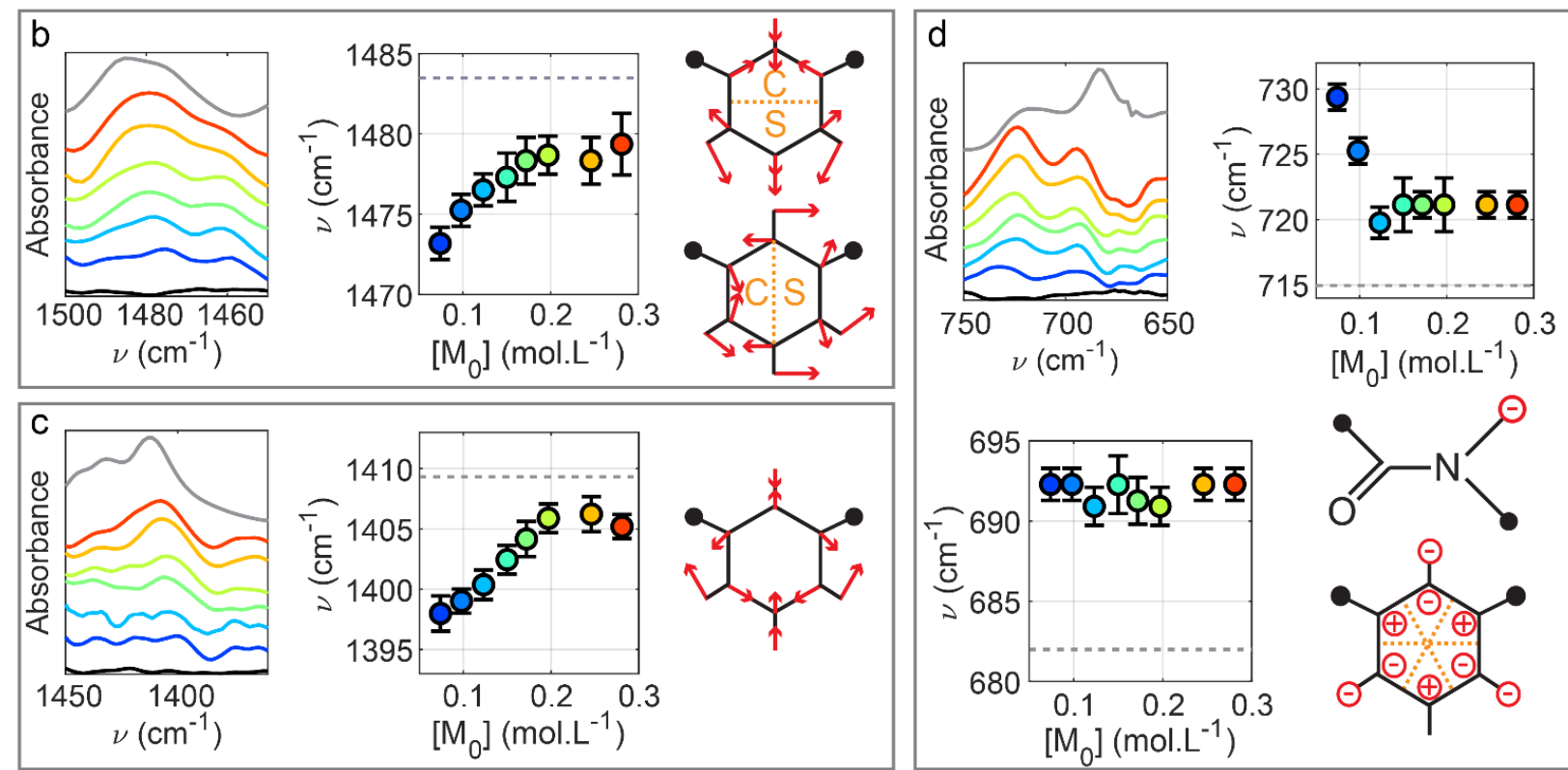

Figure 4. FTIR investigation of CNT-PMPI interaction and its dependence on ISIP monomer concentration. (a) Raw FTIR spectra of neat CNT sheets, neat PMPI, and CNT-PMPI composites obtained at various monomer concentration $\left[M_{0}\right]$. Grey boxes show wavenumbers which are represented on b, $c$ and $d$. (b-c) Zoomed view in spectral regions exhibiting peaks characteristic of in-plane vibrational modes of the meta-phenyl group of PMPI. (b) Analysis of the absorbance spectra in the 1450-1500 $\mathrm{cm}^{-1}$ range and location shift with monomer concentration of the peak characteristic of PMPI meta-phenyl ring semicircle stretch modes. The grey dashed horizontal line is placed at the location of the same vibrational peak on the neat PMPI spectrum. The C and $S$ letters are used to identify which part of the aromatic group is contracted $(C)$ and stretched $(S)$. (c) Analysis of the absorbance spectra in the 1360-1450 $\mathrm{cm}^{-1}$ range and location shift with monomer concentration of the peak characteristic of PMPI meta-phenyl ring in-plane $C$ - $H$ bendstretching mode. (d) Analysis of the absorbance spectra in the $650-750 \mathrm{~cm}^{-1}$ range and evolution with monomer concentration of out-of-plane bending vibrational mode related to the amide linkage (Amide V band) and the meta-phenyl group of PMPI. The amide linkage peak at $720 \mathrm{~cm}^{-}$ 
${ }^{1}$ for CNT-PMPI composites and $716 \mathrm{~cm}^{-1}$ is representative of the $\mathrm{N}-\mathrm{H}$ out-of-plane bending vibration while the aromatic peak at $\sim 693 \mathrm{~cm}^{-1}$ for the composites and $682 \mathrm{~cm}^{-1}$ for neat PMPI is characteristic of sextant out-of-plane bending mode of the meta-phenyl group.

To further confirm that CNT-PMPI interaction involves parallel $\pi-\pi$ stacking, we investigate the polymer's Raman-active vibrational modes in CNT-PMPI composites and compare them to that of neat PMPI. To isolate PMPI contribution from the strong CNT signal in composites, we implemented a custom principal component analysis (PCA) algorithm which enables the isolation of the partial Raman spectra (PRS, also called component spectra) involved in the overall measured signal (Figure 5) ${ }^{39}$ PCA relies on the acquisition of multiple spectra of the analyzed material (Figure 5a) which are then processed to reveal the PRS. The sum of the PRS describes the overall Raman spectrum of the characterized material. The neat CNT sheet signal is found to be accurately described with 5 components, while the CNT-PMPI composite Raman response was found to feature 6 components. Because of their characteristic peaks corresponding to the $D, G$ and $\mathrm{G}^{\prime}$ bands, ${ }^{35}$ the first 5 PRS of both the neat sheet and PMPI-CNT composite are attributed to the CNTs themselves (Figure 5b). Comparing the first 5 PRS of the CNT-PMPI composite with that of the neat CNT sheet, no significant difference is observed, and the modest deviation for components 3 and 4 can be attributed to variation of the starting CNT material as well as the difference in number of spectra acquired between the two acquisitions. The sixth component obtained for the CNT-PMPI composite is attributed to the contribution of the polymer and compared to that of neat PMPI (Figure 5c). ${ }^{34,40}$ The detailed peak assignment for the Raman signature of PMPI can be found in Table S3. The peak associated with the meta-phenyl ring trigonal ring breathing vibration (located at $1001 \mathrm{~cm}^{-1}$ ) which is also the highest intensity mode for neat PMPI completely disappear from the sixth PRS of the CNT-PMPI composite. This confirms that the polymer interacts with the CNT outer wall through parallel $\pi-\pi$ stacking which impinges the in-plane ring breathing vibration through electrostatic interaction. In parallel $\pi-\pi$ stacking, ring breathing of the meta-phenyl group is opposed by the attractive force between the positively charged groups of one aromatic group with the negatively charged $\pi$-electron-clouds of a graphene ring. This results in the annihilation of the associated vibrational peak from the PRS attributed to PMPI. 

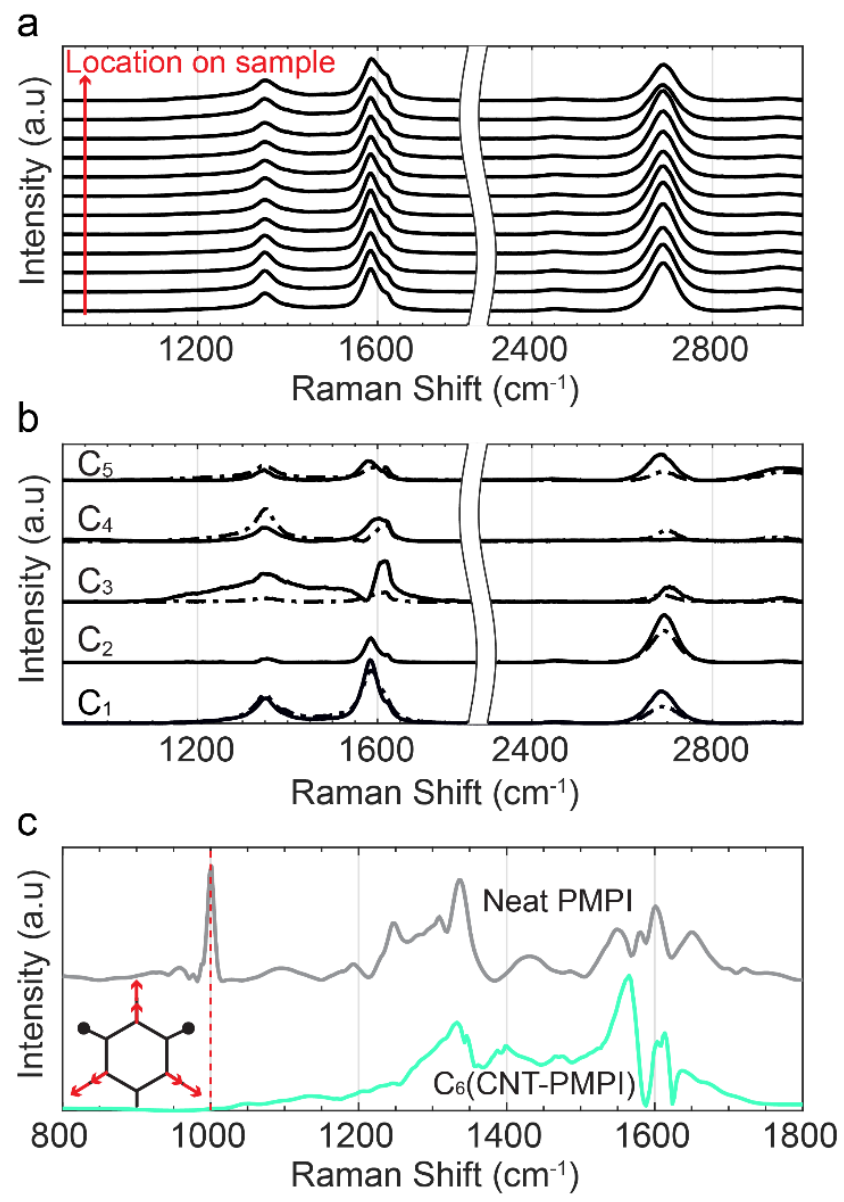

Figure 5. Raman spectroscopy investigation of CNT-PMPI interaction. (a) Representative spectra taken at different locations on the cross-section of a CNT-PMPI composite made by ISIP using a monomer concentration of 0.150 mol. $L^{-1}$. (b) First five component spectra are attributed to the CNTs in the composites. Solid lines correspond to components 1-5 extracted on the CNT-PMPI composite while dashed lines correspond to the five components picked up on the Raman response of a neat CNT sheet. (c) Sixth component spectrum obtained on the CNT-PMPI composite and comparison to the Raman spectrum of neat PMPI synthesized by interfacial-polymerization. Inset schematic represents the trigonal ring breathing mode associated with the $1000 \mathrm{~cm}^{-1}$ peak.

Most prior observations of polymer nanostructure formation in the presence of CNTs such as "shish-kebab" 41,42 or smooth sheaths ${ }^{43}$ rely on polymer crystallization through heterogeneous nucleation, as this has been observed for polyethylene, ${ }^{41}$ or poly(vinyl alcohol). ${ }^{43,44}$ Sheath formation and short-range ordering without increased crystallinity has been observed with CNTs for three other polymer systems, in addition to PMPI studied here: polycarbonate (PC), ${ }^{45}$ polyvinylidene fluoride (PVDF) ${ }^{46}$ and polypyrrole (PPy). ${ }^{47}$ All these polymers feature a flexible 
backbone and functional groups which can interact with the CNTs' outer wall through $\pi$ interactions (Figure S2). ${ }^{38,48}$ PC sheaths exhibited a multilayer structure with a strong short-range adhesion of the polymer chains onto the CNTs' surface, similarly to our observation for PMPI. ${ }^{45}$ The thickness of the polymer sheaths was only controlled in the case of PPy which was polymerized by chemical oxidation in presence of CNTs ${ }^{47}$ Both PPy polymerization and ISIP of PMPI enabled the polymer coating thickness to be varied in the 10-100 nm range by adjusting monomer concentration. However, ISIP allowed for much shorter processing times (e.g., ISIP reaction time in the order of minutes, compared to hours for chemical oxidation polymerization of PPy). Moreover, chemical oxidative polymerization limits greatly the range of available polymer systems to often electrically conductive and thermally unstable polymers. ${ }^{49}$ In contrast, ISIP offers a rapid and flexible transport-reaction scheme for the formation of CNT-based composites with chain-oriented amorphous PMPI or other suitable polycondensation polymers such as polyamides, polyesters, or polyureas. ${ }^{19}$ The high thermal stability of amorphous PMPI (up to about $400^{\circ} \mathrm{C}$ ), its superior mechanical properties, as well as its short-range ordering at the surface of the CNTs makes it particularly suitable for a broad range of high-temperature applications such as structural aerospace components, filtration membranes or high thermal conductivity materials for heat exchangers or power electronics. ${ }^{50}$

\section{CONCLUSION}

In conclusion, we demonstrated that the conformal amorphous polymer sheaths observed in CNT-meta-aramid composites produced by ISIP is the result of the interplay between polymer precipitation kinetics and parallel $\pi$ - $\pi$ stacking that involves the aromatic groups from the polymer backbone and the CNTs outer wall graphene rings. Using ReaxFF reactive force field MD simulation, we predicted that while polymer chains at the immediate vicinity of a CNT feature strong alignment and parallel $\pi-\pi$ stacking with the CNT outer wall, PMPI formed further from the nanotube is amorphous in nature. Subsequently, the formation of a smooth polymer coating around individual CNTs is shown to be a consequence of PMPI precipitation kinetics by successive heterogeneous nucleation and multilayer sheath formation. Last, we combined FT-IR and Raman spectroscopy to experimentally demonstrate the existence of both parallel $\pi-\pi$ stacking and $\mathrm{NH}-\pi$ interaction between CNTs and PMPI, as well as their decrease with increasing number of conformal layers in the meta-aramid sheath. This new understanding of CNT-polymer interaction 
combined with its implementation in the rapid and flexible ISIP synthesis scheme could guide the design and selection of polymer systems for the development and manufacturing of highperformance CNT-based composites.

\section{ASSOCIATED CONTENT}

\section{Supporting Information.}

The following files are available free of charge.

MD simulation framework, Materials and synthesis, Characterization methods, Spectroscopy and FTIR/Raman peak assignment, Comparison of PMPI obtained by ISIP with other polymer systems. (PDF)

\section{AUTHOR INFORMATION}

\section{Corresponding Authors}

*Cécile A. C. Chazot - Department of Materials Science and Engineering, Department of Mechanical Engineering, Massachusetts Institute of Technology, 77 Massachusetts Avenue, Cambridge, Massachusetts 02139, United States. Email: cchazot@mit.edu

*A. John Hart - Mechanical Engineering Department, Massachusetts Institute of Technology, 77 Massachusetts Avenue, Cambridge, Massachusetts 02139, United States. Email: ajhart@mit.edu

\section{Present Address}

$\dagger$ Behzad Damirchi - Department of Computational and Quantitative Medicine, Biomedical Research Center, City of Hope, 1218 Fifth Ave, Monrovia, CA 91016, United States

\section{Author Contributions}

C.A.C.C. and A.J.H. conceived the in-situ interfacial polymerization method. B.D. and A.C.T.D conceived the MD simulation approach. B.D. conducted the MD study. C.A.C.C designed and conducted the experiments and material characterization. C.A.C.C. wrote the first draft of the manuscript. All authors contributed to the discussion of the results and the preparation of the final version of the manuscript. C.A.C.C. coordinated the collaboration, and A.J.H. and A.C.T.D. supervised the work. 


\section{Funding Sources}

Funding was provided by the NASA Space Technology Research Institute (STRI) for UltraStrong Composites by Computational Design (US-COMP), grant NNX17AJ32G. C.A.C.C. was also supported by a 2020-2021 MathWorks Engineering Fellowship at MIT.

\section{Notes}

The authors declare no competing financial interest.

\section{ACKNOWLEDGMENT}

This work made use of the MRSEC Shared Experimental Facilities at MIT, supported by the National Science Foundation under award number DMR-1419807. This work also utilized the electron microscopy facilities at MIT.nano. The authors also thank William. J. Sawyer and Richard B. Church from MIT, for useful discussions regarding this work.

\section{ABBREVIATIONS}

ISIP, in-situ interfacial polymerization; PMPI, poly(m-phenylene isophthalamide); CNT, carbon nanotube; MD, molecular dynamic; PCFF-IFF, polymer consistent force field-interface force field; CVD, chemical vapor deposition; MPD, m-phenylene diamine; IPC, isophthaloyl chloride; SEM, Scanning Electron Microscopy; FT-IR, Fourier-transform infrared spectroscopy; PCA, principal component analysis; PRS, partial Raman spectrum/spectra.

\section{REFERENCES}

(1) Hussain, F.; Hojjati, M.; Okamoto, M.; Gorga, R. E. Review Article: Polymer-Matrix Nanocomposites, Processing, Manufacturing, and Application: An Overview. J. Compos. Mater. 2006, 40 (17), 1511-1575. https://doi.org/10.1177/0021998306067321.

(2) Eom, J.; Park, S.; Jin, H.-J.; Kwak, H. W. Multiscale Hybridization of Natural SilkNanocellulose Fibrous Composites With Exceptional Mechanical Properties. Front. Mater. 2020, 0, 98. https://doi.org/10.3389/FMATS.2020.00098.

(3) Sayed, M. M.; Mousa, H. M.; El-Aassar, M. R.; El-Deeb, N. M.; Ghazaly, N. M.; Dewidar, M. M.; Abdal-hay, A. Enhancing Mechanical and Biodegradation Properties of Polyvinyl Alcohol/Silk Fibroin Nanofibers Composite Patches for Cardiac Tissue Engineering. Mater. 
Lett. 2019, 255, 126510. https://doi.org/10.1016/J.MATLET.2019.126510.

(4) Ma, T.; Zhao, Y.; Ruan, K.; Liu, X.; Zhang, J.; Guo, Y.; Yang, X.; Kong, J.; Gu, J. Highly Thermal Conductivities, Excellent Mechanical Robustness and Flexibility, and Outstanding Thermal Stabilities of Aramid Nanofiber Composite Papers with Nacre-Mimetic Layered Structures. ACS Appl. Mater. Interfaces 2019, 12 (1), 1677-1686. https://doi.org/10.1021/ACSAMI.9B19844.

(5) Fan, J.; Wang, J.; Shi, Z.; Yu, S.; Yin, J. Kevlar Nanofiber-Functionalized Multiwalled Carbon Nanotubes for Polymer Reinforcement. Mater. Chem. Phys. J. 2013, 141, 861-868. https://doi.org/10.1016/j.matchemphys.2013.06.015.

(6) Chazot, C. A. C.; Hart, A. J. Understanding and Control of Interactions between Carbon Nanotubes and Polymers for Manufacturing of High-Performance Composite Materials.

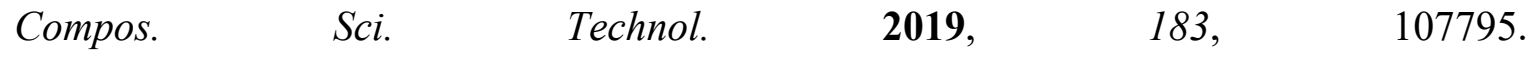
https://doi.org/10.1016/J.COMPSCITECH.2019.107795.

(7) Liu, Y.; Kumar, S. Polymer / Carbon Nanotube Nano Composite Fibers - A Review. 2014. https://doi.org/10.1021/am405136s.

(8) Guan, Y.; Li, W.; Zhang, Y.; Shi, Z.; Tan, J.; Wang, F.; Wang, Y. Aramid Nanofibers and Poly (Vinyl Alcohol) Nanocomposites for Ideal Combination of Strength and Toughness via Hydrogen Bonding Interactions. Compos. Sci. Technol. 2017, 144, 193-201. https://doi.org/10.1016/j.compscitech.2017.03.010.

(9) Minus, M. L.; Chae, H. G.; Kumar, S. Interfacial Crystallization in Gel-Spun Poly ( Vinyl Alcohol )/ Single-Wall Carbon Nanotube Composite Fibers. Macromol. Chem. Phys. 2009, 210, 1799-1808. https://doi.org/10.1002/macp.200900223.

(10) Wardle, B. L.; Saito, D. S.; Garcia, E. J.; Hart, A. J.; Guzman De Villoria, R.; Verploegen, E. A. Fabrication and Characterization of Ultrahigh-Volume-Fraction Aligned Carbon Nanotube-Polymer Composites. Adv. Mater. 2008, 20 (14), 2707-2714. https://doi.org/10.1002/adma.200800295.

(11) De Volder, M. F. L.; Tawfick, S. H.; Baughman, R. H.; Hart, A. J. Carbon Nanotubes: 
Present and Future Commercial Applications. Science (80-. ). 2013, 339 (6119), 535-539. https://doi.org/10.1126/science.1222453.

(12) Davijani, A. A. B.; Kumar, S. Ordered Wrapping of Poly ( Methyl Methacrylate ) on Single Wall Carbon Nanotubes. Polymer (Guildf). 2015, 70, 278-281. https://doi.org/10.1016/j.polymer.2015.06.018.

(13) Tallury, S. S.; Pasquinelli, M. A. Molecular Dynamics Simulations of Polymers with Stiff Backbones Interacting with Single-Walled Carbon Nanotubes. J. Phys. Chem. B 2010, 114, 9349-9355. https://doi.org/10.1021/jp908001d.

(14) Jolowsky, C.; Sweat, R.; Park, J. G.; Hao, A.; Liang, R. Microstructure Evolution and SelfAssembling of CNT Networks during Mechanical Stretching and Mechanical Properties of Highly Aligned CNT Composites. Compos. Sci. Technol. 2018, 166, 125-130. https://doi.org/10.1016/J.COMPSCITECH.2018.04.003.

(15) Cividanes, L. S.; Simonetti, E. A. N.; Moraes, M. B.; Fernandes, F. W.; Thim, G. P. Influence of Carbon Nanotubes on Epoxy Resin Cure Reaction Using Different Techniques: A Comprehensive Review. Polym. Eng. Sci. 2014, 54, 2461-2469. https://doi.org/10.1002/pen.

(16) Zhang, S.; Hao, A.; Nguyen, N.; Oluwalowo, A.; Liu, Z.; Dessureault, Y.; Park, J. G.; Liang, R. Carbon Nanotube/Carbon Composite Fiber with Improved Strength and Electrical Conductivity via Interface Engineering. Carbon N. Y. 2019, 144, 628-638. https://doi.org/10.1016/J.CARBON.2018.12.091.

(17) Coleman, J. N.; Blau, W. J.; Dalton, A. B.; Muñoz, E.; Collins, S.; Kim, B. G.; Selvidge, M.; Vieiro, G.; Baughman, R. H.; Collins, S.; Kim, B. G.; Razal, J.; Selvidge, M.; Dalton, A. B.; Mun, E.; Vieiro, G.; Baughman, R. H. Improving the Mechanical Properties of Single-Walled Carbon Nanotube Sheets by Intercalation of Polymeric Adhesives. Appl. Phys. Lett. 2003, 82. https://doi.org/10.1063/1.1559421.

(18) Zhang, M.; Atkinson, K. R.; Baughman, R. H. Multifunctional Carbon Nanotube Yarns by Downsizing an Ancient Technology. Science (80-. ). 2004, 306, 1358-1362. 
(19) Morgan, P. W.; Kwolek, S. L. Interfacial Polycondensation. II. Fundamentals of Polymer Formation at Liquid Interfaces. J. Polym. Sci. 1959, 40 (September 1958), 299-327.

(20) Chazot, C. A. C.; Jons, C. K.; Hart, A. J. In Situ Interfacial Polymerization: A Technique for Rapid Formation of Highly Loaded Carbon Nanotube-Polymer Composites. Adv. Funct. Mater. 2020, 30 (52), 2005499. https://doi.org/10.1002/adfm.202005499.

(21) Kakida, H.; Chatani, Y.; Tadokoro, H. Crystal Structure of Poly(m-Phenylene Isophthalamide). J. Polym. Sci. Polym. Phys. Ed. 1976, 14 (3), 427-435. https://doi.org/10.1002/POL.1976.180140305.

(22) Yang, Z.; Wang, Z.; Tian, X.; Xiu, P.; Zhou, R. Amino Acid Analogues Bind to Carbon Nanotube via $\pi-\pi$ Interactions: Comparison of Molecular Mechanical and Quantum Mechanical Calculations. J. Chem. Phys. 2012, $136 \quad$ (2), 025103. https://doi.org/10.1063/1.3675486.

(23) Hermans, P. H.; Platzek, P. Beiträge Zur Kenntnis Des Deformationsmechanismus Und Der Feinstruktur Der Hydratzellulose. Kolloid-Zeitschrift 1939, $88 \quad$ (1), 68-72. https://doi.org/10.1007/BF01518890.

(24) Senftle, T. P.; Hong, S.; Islam, M. M.; Kylasa, S. B.; Zheng, Y.; Shin, Y. K.; Junkermeier, C.; Engel-Herbert, R.; Janik, M. J.; Aktulga, H. M.; Verstraelen, T.; Grama, A.; Van Duin, A. C. T. The ReaxFF Reactive Force-Field: Development, Applications and Future Directions. npj Comput. Mater. 2016, 2 (September 2015). https://doi.org/10.1038/npjcompumats.2015.11.

(25) Dasgupta, N.; Yilmaz, D. E.; Van Duin, A. Simulations of the Biodegradation of CitrateBased Polymers for Artificial Scaffolds Using Accelerated Reactive Molecular Dynamics. J. Phys. Chem. B 2020, 124 (25), 5311-5322. https://doi.org/10.1021/acs.jpcb.0c03008.

(26) Vashisth, A.; Ashraf, C.; Bakis, C. E.; van Duin, A. C. T. Effect of Chemical Structure on Thermo-Mechanical Properties of Epoxy Polymers: Comparison of Accelerated ReaxFF Simulations and Experiments. Polymer (Guildf). 2018, 158 (November), 354-363. https://doi.org/10.1016/j.polymer.2018.11.005. 
(27) Van Duin, A. C. T.; Dasgupta, S.; Lorant, F.; Goddard, W. A. ReaxFF: A Reactive Force Field for Hydrocarbons. J. Phys. Chem. A 2001, 105 (41), 9396-9409. https://doi.org/10.1021/jp004368u.

(28) Brenner, D. W. Empirical Potential for Hydrocarbons for Use in Simulating the Chemical Vapor Deposition of Diamond Films. Phys. Rev. B 1990, 42 (15), 9458-9471. https://doi.org/10.1103/PhysRevB.42.9458.

(29) Damirchi, B.; Radue, M.; Kanhaiya, K.; Heinz, H.; Odegard, G. M.; Van Duin, A. C. T. ReaxFF Reactive Force Field Study of Polymerization of a Polymer Matrix in a Carbon Nanotube-Composite System. J. Phys. Chem. C 2020, 124. https://doi.org/10.1021/acs.jpcc.0c03509.

(30) Meng, X.; Zhang, B.; Li, H.; Li, F.; Kang, Z.; Li, M.; Chen, Y. A Theoretical Analysis on Self-Collapsing of Nanotubes. Int. J. Solids Struct. 2019, 160, 51-58. https://doi.org/10.1016/J.IJSOLSTR.2018.10.016.

(31) Cheng, Q.; Li, M.; Jiang, L.; Tang, Z. Bioinspired Layered Composites Based on Flattened Double-Walled Carbon Nanotubes. Adv. Mater. 2012, 24 (14), 1838-1843. https://doi.org/10.1002/ADMA.201200179.

(32) Tang, T.; Jagota, A.; Hui, C.-Y.; Glassmaker, N. J. Collapse of Single-Walled Carbon Nanotubes. J. Appl. Phys. 2005, 97 (7), 074310. https://doi.org/10.1063/1.1883302.

(33) Qian, W.; Wei, F.; Liu, T.; Wang, Z.; Li, Y. What Causes the Carbon Nanotubes Collapse in a Chemical Vapor Deposition Process. J. Chem. Phys. 2002, 118 (2), 878. https://doi.org/10.1063/1.1527897.

(34) Lin-Vien, D.; Colthup, N. B.; Fateley, W. G.; Grasselli, J. G. The Handbook of Infrared and Raman Characteristic Frequencies of Organic Molecules, Academic Press, INC.; Harcourt Brace Jovanovich, Publishers: San Diego, 1991.

(35) Lefrant, S.; Baibarac, M.; Baltog, I. Raman and FTIR Spectroscopy as Valuable Tools for the Characterization of Polymer and Carbon Nanotube Based Composites. J. Mater. Chem. 2009, 19 (32), 5690-5704. https://doi.org/10.1039/B821136A. 
(36) Villar-Rodil, S.; Paredes, J. I.; Martinez-Alonso, A.; Tascón, J. M. D. Atomic Force Microscopy and Infrared Spectroscopy Studies of the Thermal Degradation of Nomex Aramid Fibers. Chem. Mater. 2001, 13 (11), 4297-4304. https://oi.org/10.1021/cm001219f.

(37) Yang, D. Q.; Rochelte, J. F.; Sacher, E. Spectroscopic Evidence for $\pi-\pi$ Interaction between Poly(Diallyl Dimethylammonium) Chloride and Multiwalled Carbon Nanotubes. J. Phys. Chem. B 2005, 109 (10), 4481-4484. https://doi.org/10.1021/jp044511+.

(38) Baskaran, D.; Mays, J. W.; Bratcher, M. S. Noncovalent and Nonspecific Molecular Interactions of Polymers with Multiwalled Carbon Nanotubes. Chem. Mater. 2005, 17 (13), 3389-3397. https://doi.org/10.1021/cm047866e.

(39) Woelffel, W.; Claireaux, C.; Toplis, M. J.; Burov, E.; Barthel, É.; Shukla, A.; Biscaras, J.; Chopinet, M. H.; Gouillart, E. Analysis of Soda-Lime Glasses Using Non-Negative Matrix Factor Deconvolution of Raman Spectra. J. Non. Cryst. Solids 2015, 428, 121-131. https://doi.org/10.1016/J.JNONCRYSOL.2015.08.016.

(40) Edwards, H. G. M.; Hakiki, S. Raman Spectroscopic Studies of Nomex and Kevlar Fibres under Stress. Br. Polym. J. 1989, 21 (6), 505-512. https://doi.org/10.1002/PI.4980210611.

(41) Zhang, S.; Lin, W.; Yu, X.; Wong, C. P.; Cheng, S. Z. D.; Bucknall, D. G. Surface-Induced Polymer Crystallization in High Volume Fraction Aligned Carbon Nanotube-Polymer Composites. Macromol. Chem. Phys. 2010, 211 (9), 1003-1011. https://doi.org/10.1002/macp.200900686.

(42) Li, L.; Li, B.; Hood, M. A.; Li, C. Y. Carbon Nanotube Induced Polymer Crystallization: The Formation of Nanohybrid Shish-Kebabs. Polymer (Guildf). 2009, 50 (4), 953-965. https://doi.org/10.1016/j.polymer.2008.12.031.

(43) Dassios, K. G. Poly(Vinyl Alcohol)-Infiltrated Carbon Nanotube Carpets. Mater. Sci. Appl. 2012, 03 (09), 658-663. https://doi.org/10.4236/msa.2012.39096.

(44) Minus, M. L.; Chae, H. G.; Kumar, S. Single Wall Carbon Nanotube Templated Oriented Crystallization of Poly ( Vinyl Alcohol ). Polymer (Guildf). 2006, 47, 3705-3710. 
https://doi.org/10.1016/j.polymer.2006.03.076.

(45) Ding, W.; Eitan, A.; Fisher, F. T.; Chen, X.; Dikin, D. A.; Andrews, R.; Brinson, L. C.; Schadler, L. S.; Ruoff, R. S. Direct Observation of Polymer Sheathing in Carbon NanotubePolycarbonate Composites. Nano Lett. 2003, 3 (11), 1593-1597. https://doi.org/10.1021/nl0345973.

(46) Tran, M. Q.; Cabral, J. T.; Shaffer, M. S. P.; Bismarck, A. Direct Measurement of the Wetting Behavior of Individual Carbon Nanotubes by Polymer Melts: The Key to Carbon Nanotube - Polymer Composites. Nano Lett. 2008, 8 (9), 2744-2750. https://doi.org/10.1021/nl801209g.

(47) Zhang, B.; Xu, Y.; Zheng, Y.; Dai, L.; Zhang, M.; Yang, J.; Chen, Y.; Chen, X.; Zhou, J. A Facile Synthesis of Polypyrrole/Carbon Nanotube Composites with Ultrathin, Uniform and Thickness-Tunable Polypyrrole Shells. Nanoscale Res. Lett. 2011, 6 (1), 1-9. https://doi.org/10.1186/1556-276X-6-431.

(48) Politzer, P.; Murray, J. S.; Clark, T. Halogen Bonding: An Electrostatically-Driven Highly Directional Noncovalent Interaction. Phys. Chem. Chem. Phys. 2010, 12 (28), 7748-7757. https://doi.org/10.1039/c004189k.

(49) Higashimura, H.; Kobayashi, S. Oxidative Polymerization. Encycl. Polym. Sci. Technol. 2016, 1-37. https://doi.org/10.1002/0471440264.PST226.PUB2.

(50) Singh, V.; Bougher, T. L.; Weathers, A.; Cai, Y.; Bi, K.; Pettes, M. T.; McMenamin, S. A.; Lv, W.; Resler, D. P.; Gattuso, T. R.; Altman, D. H.; Sandhage, K. H.; Shi, L.; Henry, A.; Cola, B. A. High Thermal Conductivity of Chain-Oriented Amorphous Polythiophene. Nat. Nanotechnol. 2014, 9 (5), 384-390. https://doi.org/10.1038/nnano.2014.44. 